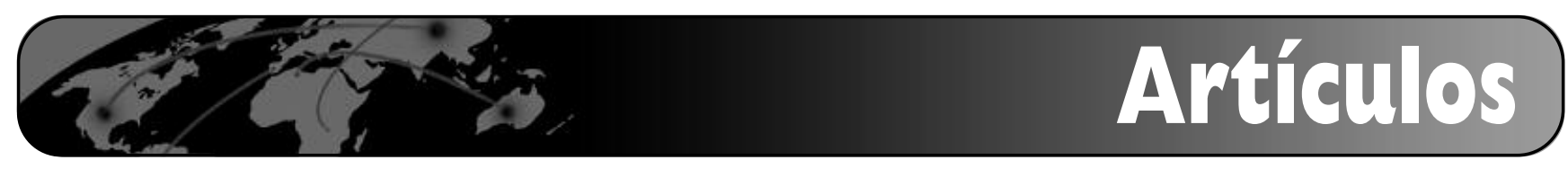

\title{
Aplicación de técnicas de visualización basadas en co-citaciones a los estudios cinematográficos
}

\author{
Por Carlos Leal Zubiete
}

\begin{abstract}
Resumen: La aplicación de técnicas de visualización de información al análisis cuantitativo de citas en documentos científicos se ha demostrado una herramienta muy útil para revelar estructuras y tendencias de investigación dentro de las distintas áreas de conocimiento. Partiendo del concepto de cita cinematográfica, entendida como un guiño intertextual que se integra en el flujo de la narración audiovisual, se propone una aproximación similar para detectar patrones de influencia entre textos fílmicos. A partir de una representación visual de los datos de co-citación entre películas del Hollywood clásico extraídos de la base de datos

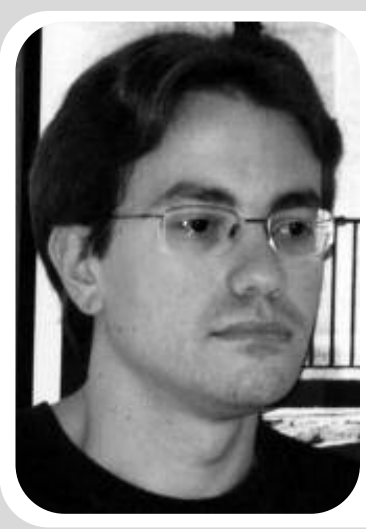
Carlos Leal es licenciado en periodismo por la Universidad de Sevilla y master en información y documentación por la Universidad Carlos III de Madrid, centro en el que está realizando el programa de doctorado en documentación. Trabaja en el ámbito de la gestión de contenidos en el Portal del Ciudadano de la Junta de Andalucía y edita la revista digital de información cinematográfica Cinestrenos.com.

http://andaluciajunta.es/

http://www.cinestrenos.com
\end{abstract} IMDb, se ilustra la utilidad de esta técnica para los es-

tudios cinematográficos en general y la teoría de géneros en particular.

Palabras clave: Cita cinematográfica, Co-citación, Teoría de géneros cinematográficos, Intertextualidad, Visualización de información.

\section{Title: An application of co-citation based visualisation techniques in the field of Film Studies}

Abstract: The application of information visualisation techniques to quantitative citation analysis in scientific documents has proved very useful in revealing hidden structures and research trends in several disciplines. A similar approach is proposed in the field of Film Studies based on the concept of «filmic citation», understood as an allusion to a different audiovisual text that blends seamlessly into the flow of the cinematographic narrative. Thus, this technique allows the detection of patterns of influence between audiovisual texts. A visual representation of the co-citation data for Classic Hollywood films extracted from the IMDb database illustrates the utility of this technique for Film Studies and, particularly, Film Genre Studies.

Keywords: Filmic citation, Co-citation, Film genre theory, Intertextuality, Information visualisation.

Leal Zubiete, Carlos. «Aplicación de técnicas de visualización basadas en co-citaciones a los estudios cinematográficos». En: El profesional de la información, 2006, julio-agosto, v. 15, n. 4, pp. 251-257.

\section{Introducción}

El lenguaje cinematográfico ha mostrado desde sus orígenes una amplia tendencia a la autorreflexividad, un fenómeno cuya principal muestra es el rápido contagio de ideas y recursos expresivos entre textos fílmicos. Como consecuencia de esta actividad referencial, a lo largo de la historia han surgido patrones en ocasiones fuertemente codificados, que configuran el objeto de estudio de la teoría de géneros. La manifestación más evidente de la importancia de la intertextualidad en el cine contemporáneo es la proliferación de la cita cinematográfica, entendida como una remisión a un texto fílmico anterior que se incorpora al discurso audiovisual a modo de homenaje o comentario sobre los temas subyacentes a la narración.

La tesis principal de este artículo es que el estudio cuantitativo de las citas cinematográficas ofrece una información relevante para contrastar la pertinencia de los principales modelos conceptuales manejados por los estudios cinematográficos, particularmente la teoría de géneros, al poner de manifiesto patrones subyacentes de influencia entre textos fílmicos. La inspira- 
ción básica proviene de los estudios de visualización de disciplinas por medio del recuento de co-citaciones realizados por autores como Small, White y McCain, que emplean técnicas de análisis de redes y escalamiento multidimensional (MDS) ${ }^{1}$ para representar gráficamente la estructura de un área del conocimiento científico.

\section{Concepto de cita cinematográfica}

Ya desde su desarrollo en las primeras décadas del siglo XX, el lenguaje cinematográfico ha demostrado un componente elevado de autoconsciencia y preocupación por los aspectos formales de la narración audiovisual. Sin embargo, no fue hasta la década de los 60 cuando el análisis de la intertextualidad se convirtió en una de las cuestiones prioritarias en el ámbito de los estudios cinematográficos. Dos circunstancias contribuyeron al desarrollo de esta preocupación: el nacimiento de una nueva cultura cinematográfica entre los espectadores, facilitada por la televisión y las filmotecas, y la aparición de una generación de realizadores con formación universitaria y una mayor preocupación tanto por el lenguaje como por la historia del cine (Scorsese, Allen, Coppola, etc.).

\section{«Desde los años 60 la cita cinematográfica se ha convertido en un elemento expresivo esencial que los autores usan para hacer comentarios sobre el espacio ficcional de sus películas»}

De esta forma, según explica Noël Carroll (1998), desde los años 60 la alusión a la historia del cine se ha convertido en una figura expresiva fundamental que los autores utilizan para hacer comentarios sobre el espacio de ficción creado por sus películas. Estas referencias toman formas diversas, que pueden ser la imitación directa de modelos cinematográficos, la inserción de fragmentos de filmes, la mención de películas y directores en el diálogo, la inclusión de títulos en las marquesinas de los cines, posters y otros elementos del decorado, etc. Para este autor, la gran cantidad de directores que hacen de la alusión un elemento nuclear de su visión autorial convierte a este fenómeno en la estructura simbólica más significativa que marca la transición entre el cine clásico y el contemporáneo.

La mayor parte de los trabajos teóricos en torno a la intertextualidad cinematográfica sugieren adaptaciones de estudios provenientes de la literatura o el arte. Así, Stam (2001) aplica al mundo del cine el concepto de transtextualidad de Gérard Genette, que tra-

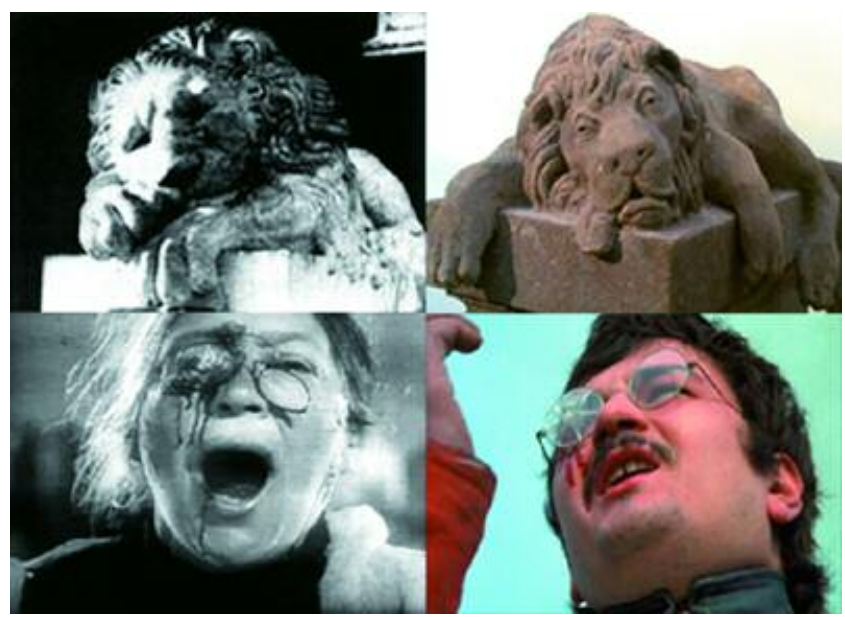

Figura 1

ta de recoger todos los tipos de relaciones, manifiestas o secretas, que pueden producirse entre dos textos. En concreto, Stam define la intertextualidad (en sentido estricto) como la copresencia efectiva de dos textos bajo la forma de cita, referencia o plagio. Aplicado al mundo del cine, una cita es el equivalente a la inclusión de un fragmento de un filme dentro de otro (p. ej. el clip de El hombre tranquilo en E.T. el extraterrestre). Por su parte, la referencia es una evocación auditiva o visual de un texto fílmico diferente. La figura 1 muestra dos ejemplos de referencia al clásico de Eisenstein El acorazado Potemkin (1925), extraídos del filme de Woody Allen La última noche de Boris Grushenko (1975).

El concepto de intertextualidad definido de esta forma equivale esencialmente a lo que en este trabajo entenderemos como cita cinematográfica. Mijail Iampolski (1996) profundiza en las implicaciones de este fenómeno al definir la cita cinematográfica como «un fragmento de texto que perturba el desarrollo lineal de este último y que recibe fuera del texto una motivación que lo integra en él». Destaca en esta definición el carácter significativo que confiere necesariamente a la cita cinematográfica. Dado que interrumpe el discurso natural del filme aportando elementos de reflexión que deben ser tenidos en cuenta en la interpretación del texto, no es procedente hablar de citas 'arbitrarias' o 'caprichosas'.

Una cuestión problemática que suscita esta definición es que, si bien es evidente que desde un punto de vista analítico las citas perturban el desarrollo del texto original, en la mayoría de los casos éstas se integran dentro del flujo narrativo del filme de una forma transparente para el espectador no informado. El resultado, como explica Carroll (1998), es un sistema de comunicación doble en el que uno de los discursos (el que pone en relación el texto fílmico con sus referentes anteriores en la historia del cine) es inteligible únicamente por una parte determinada de la audien- 
cia: «la viabilidad de los géneros es la que permite a la alusión convertirse en una opción práctica. El director preocupado por la historia del cine puede habilidosamente manipular las formas antiguas, satisfaciendo a la clientela adolescente mientras que convenientemente envía alusiones a los habituales cinéfilos de las primeras filas».

\section{«La cita cinematográfica interrumpe el discurso natural del filme aportando elementos de reflexión que deben ser tenidos en cuenta en la interpretación»}

De hecho, se espera del público informado no sólo que sea capaz de identificar las referencias a películas, autores, géneros, etc., planteadas por el texto fílmico, sino también que reconozca las variaciones que se producen sobre esas mismas (ritmos, personajes, líneas argumentales, etc.). Como explica Noël Carroll, dentro del cine popular estas desviaciones son las que constituyen el sello personal del autor.

\section{Revisión de la teoría de géneros cinematográficos}

El sistema de los géneros es uno de los primeros constructos teóricos que se desarrollaron en el campo de los estudios cinematográficos, y su utilidad ha sido indudable al analizar los procesos de producción, distribución y consumo de textos fílmicos, particularmente en la época del Hollywood clásico. En su concepción tradicional equivalen a categorías analíticas que agrupan filmes con un grado de especialización elevado, que implica convenciones temáticas y formales fácilmente identificables por el espectador.

Los elementos que determinan qué filmes se adscriben a un determinado género son variables. Como explica Coursodon (1996), algunos géneros establecen límites espaciales y temporales para la acción (western), mientras que otros se definen por el uso de determinadas técnicas (animación) o medios estilísticos (la música diegética ${ }^{2}$ en el musical), o incluso por los efectos sobre el espectador (terror). Esta disparidad de criterios permite la existencia de películas que cumplen los criterios de más de una categoría, como el western musical La leyenda de la ciudad sin nombre (Logan, 1968).

Muchos investigadores han puesto de manifiesto las limitaciones teóricas que conlleva una delimitación tan imprecisa. Así, Rick Altman (2003) propone un acercamiento semántico/sintáctico al cine de género, que tenga en cuenta al mismo tiempo los elementos identificadores (características, actitudes, personajes, planos, lugares, escenarios) y las relaciones esenciales entre elementos variables que constituyen la sintaxis fundamental del género: «aceptando simultáneamente las nociones semánticas y sintácticas de género nos proveemos de una forma de tratar críticamente distintos niveles de genericidad. Además, un acercamiento dual permite una descripción más precisa de las numerosas conexiones intergenéricas con frecuencia ignoradas por las aproximaciones únicas».

Otros investigadores llegan a cuestionar la validez del concepto de género. Janet Steiger (2003) analiza la pretendida pureza de los géneros en el Hollywood clásico y concluye que se trata de un lugar común con escaso anclaje en la realidad, sustentado por estudios que emplean métodos críticos que privilegian la coherencia y la uniformidad. Esta autora propone sustituir el concepto de género por el de patrón, que es un elemento crucial para los procesos de comparación y, por lo tanto, para el análisis de la desviación, el diálogo y la crítica. "Aunque las prácticas de agrupar películas por géneros hayan sido eclécticas, el agrupamiento de películas puede seguir siendo una actividad crítica importante por cuanto que puede arrojar luz sobre lo que hacen los productores y consumidores de cine», escribe Steiger.

En todo caso, ya sea desde una perspectiva integradora del concepto de género que contemple las relaciones intergenéricas, como propone Altman, o bien desde el rechazo de este concepto y su sustitución por el menos específico de patrón, como sugiere Steiger, se hace evidente la necesidad de establecer esquemas semióticos que faciliten el análisis y el agrupamiento de textos fílmicos. Dado que la construcción real de estas estructuras se produce necesariamente por un proceso de influencia, de contagio entre películas o movimientos cinematográficos, el análisis de la intertextualidad fílmica se erige como un proceso fundamental para la identificación de estos patrones.

\section{«El análisis cuantitativo de las citas cinematográficas ofrece información relevante para contrastar los modelos teóricos desarrollados en los estudios cinematográficos»}

En paralelo a los necesarios estudios con metodologías cualitativas que quedan por hacer en este ámbito, es la tesis central de esta investigación que un análisis cuantitativo de la intertextualidad fílmica a través de su manifestación más tangible, la cita cinematográ- 
fica, ofrece información relevante para contrastar la pertinencia de los modelos teóricos desarrollados por los estudios cinematográficos.

\section{Principios de la investigación}

La inspiración básica parte de los trabajos de visualización de información de autores como Stuart Card o Chaomei Chen. En el ámbito de la documentación, los estudios de visualización de información han confluido con una de las líneas principales de investigación de la disciplina, el análisis cuantitativo de las citas en documentos científicos.

El punto de partida de este tipo de estudios es que el análisis de la forma en que los documentos científicos se citan unos a otros pone de relevancia tendencias, grupos de interés y campos de investigación prioritarios, hasta el punto de que se puede construir un mapa de la producción científica que sirva de orientación a los sociólogos e historiadores de la ciencia para contrastar las conclusiones obtenidas con metodologías cualitativas.

Una de las medidas más empleadas para la visualización de literaturas científicas es la co-citación, concepto introducido por Small en 1973. Se considera que dos documentos científicos han sido co-citados cuando aparecen juntos en la bibliografía de un tercer documento; según sea mayor este índice entre dos documentos (cuantas más ocasiones han sido citados juntos en bibliografías) más estrecha es la conexión intelectual entre ellos desde la percepción de la comunidad científica. Con posterioridad, los datos recopilados se representan gráficamente mediante técnicas de escalamiento multidimensional $(M D S)$ o similares.

Trabajos de este tipo han sido realizados con éxito en ámbitos variados como las ciencias de la naturaleza (Small; Griffith, 1974), la bioquímica y biología molecular (Garfield, 1981) o la documentación (White; McCain, 1998). Sin embargo, hasta el momento no se han desarrollado esfuerzos similares en el mundo del cine; el único precedente indirecto de esta investigación es el trabajo de J. A. Walter (2004), que construye un mapa de películas a partir de información obtenida de críticas cinematográficas para ilustrar la técnica de representación $H-M D S$.

\section{Metodología y fuentes}

Con el fin de contrastar la efectividad de la técnica propuesta, en el siguiente apartado expondremos una representación visual de los datos de co-citación de textos fílmicos del Hollywood clásico, extraídos de la base de datos Internet Movie Database (en adelante $I M D b)$. Esta exhaustiva fuente de información contiene fichas de más de 420.000 películas (cortometrajes y largometrajes) y, en menor medida, otros productos audiovisuales como series de televisión o videojuegos. Recoge específicamente los vínculos que se producen entre filmes dentro de la categoría movie connections. En concreto, contempla tres tipos de relaciones que se ajustan al concepto de cita cinematográfica expuesto: "references" (referencia), "features" (inclusión de un fragmento) y "spoofs" (parodia).

\section{«La base de datos Internet \\ Movie Database recoge los vínculos que se producen entre películas dentro de la categoría 'movie connections'”}

$I M D b$ es la única fuente de datos que ofrece información sobre citas cinematográficas con suficiente exhaustividad y rigor como para permitir un análisis cuantitativo con ciertas garantías. Para la obtención de la información, se basa sobre todo en las aportaciones de sus usuarios, que son posteriormente revisadas por un equipo de especialistas. En la actualidad, ésta parece la única vía razonable para recopilar la información de citaciones. Aunque las técnicas automatizadas de reconocimiento de imágenes y sonido podrían ayudar a identificar algunos casos de referencias, la abrumadora cantidad de información que sería necesario procesar hace de éste un acercamiento poco realista.

La elección del marco espacio-temporal concreto de la representación que expondremos a continuación (el cine de Hollywood en la época clásica) responde a que es, sin duda, el período más estudiado por la teoría de géneros, y en el que este paradigma analítico ha mostrado una utilidad mayor. Para la extracción de los datos de co-citación se han considerado todas las películas producidas en EUA en los años que transcurren desde 1929 (consolidación del cine sonoro) a 1959 (estreno de Ben-Hur, la última superproducción rodada bajo el sistema de los estudios). La consulta, formulada a la base de datos el 25 de enero de 2006, devuelve un conjunto de 24.527 filmes, aunque no todos tienen datos de citación disponibles.

De acuerdo con lo expuesto en el punto anterior, hemos considerado que dos películas han sido co-citadas cada vez que un tercer filme alude a ambas a través de cualquiera de las formas mencionadas (referencia, inclusión o parodia). Así pues, el valor de co-citación entre dos películas determinadas equivale al número de títulos posteriores que las incluyen en común entre sus referentes. Cabe destacar que, aunque la representación contempla únicamente películas rodadas en el período clásico de Hollywood, los datos de cita- 


\begin{tabular}{|l|r|r|r|r|r|}
\hline & \multicolumn{1}{|c|}{ 2 } & \multicolumn{1}{c|}{3} & \multicolumn{1}{c|}{4} & \multicolumn{1}{c|}{5} \\
\hline 1. The wizard of Oz (1939) & 0 & 34 & 38 & 25 & 24 \\
\hline 2. Casablanca (1942) & 34 & 0 & 39 & 14 & 15 \\
\hline 3. Gone with the wind (1939) & 38 & 39 & 0 & 11 & 21 \\
\hline 4. Frankenstein (1931) & 25 & 14 & 11 & 0 & 19 \\
\hline 5. King Kong (1933) Tabla 1 & 24 & 15 & 21 & 19 & 0 \\
\hline
\end{tabular}

ción se han extraído de todos los textos audiovisuales de $I M D b$.

El recuento de los casos de co-citación se ha realizado automáticamente a partir de las listas fuente de $I M D b^{4}$. Para la extracción de los datos, se ha desarrollado un algoritmo en el lenguaje $A W K$ que recorre la lista y extrae los casos de co-citación entre las películas especificadas en el input, almacenando los resultados en una matriz de doble entrada. La tabla 1 muestra los datos de las cinco películas con mayor índice de co-citación.

\section{«La representación visual de los datos de co-citación de películas del Hollywood clásico muestra hasta qué punto la concepción genérica del cine está asentada en la visión de los autores»}

La representación que se reproduce en el siguiente apartado está elaborada con el programa Pajek ${ }^{5}$, diseñado para el análisis y la visualización de grandes redes. En concreto, para la distribución espacial de los nodos se ha empleado el algoritmo desarrollado por Fructerman y Reingold en dos dimensiones, que cumple los criterios estéticos más usuales en este tipo de visualizaciones.

Con el fin de simplificar lo más posible la representación gráfica de los datos de co-citación extraídos, no se han considerado pertinentes los vínculos cuyo valor es inferior a 7. Asimismo, se han excluido todos los nodos cuyo grado es inferior a 3, es decir, aquellos que han sido co-citados con dos películas o menos.

\section{Resultados y análisis}

A través de la representación visual de los datos de co-citación pretendemos analizar hasta qué punto la concepción genérica de la historia del cine está asentada en la percepción de los autores. Un gráfico con clusters claramente definidos y escasamente conectados entre sí indicaría que la idea de género es algo más que un paradigma analítico más o menos afortunado; por el contrario, una red muy tupida e indiferenciada apoyaría la hipótesis de Steiger sobre la artificialidad del concepto de género cinematográfico.

La impresión visual que se obtiene de la representación, reproducida en la figura 2 , respalda la concepción genérica del cine clásico de Hollywood. A simple vista se pueden identificar seis clusters bien diferenciados, que pueden adscribirse a géneros tradicionalmente reconocidos por los historiadores del cine:

-Musical.

—Animación.

—Ciencia-ficción.

-Terror.

—Cine negro/thriller.

-Western.

Los géneros que en el mapa aparecen reflejados con una mayor nitidez son aquellos que presentan una mayor especialización narrativa y unos códigos semióticos más fuertes. Además, cabe destacar que los clusters agotan plenamente las películas genéricas de la selección; el único caso de desplazamiento observable es el del western El tesoro de Sierra Madre (Huston, 1948), que se sitúa en posiciones cercanas al cine negro. Tres factores pueden haber influido en este agrupamiento irregular: la autoría del filme (está dirigido por John Huston, al igual que El halcón maltés), las estrellas (el protagonista es Humphrey Bogart, el actor más conocido del género) y ciertas características del propio filme. Y es que, si desde el punto de vista semántico hay sobrados elementos para clasificar $E l$ tesoro de Sierra Madre como un western (se desarrolla en el far west con el tema de los buscadores de oro como trasfondo), atendiendo a la sintaxis del filme se observan elementos comunes con el cine negro: la codicia como motor de la acción, el tono pesimista, etc.

Los enlaces intergenéricos son relativamente infrecuentes; únicamente las películas más populares de un determinado género tienden a ser citadas junto a películas centrales de otros. Dentro de cada género, las posiciones más cercanas al centro del gráfico están ocupadas por películas que abren lenguajes cinematográficos y establecen un canon; así sucede en el caso del cine de terror (Frankenstein, Drácula), la animación (Blancanieves y los siete enanitos), el musical (Cantando bajo la lluvia) o el western (Solo ante el peligro).

La posición relativa de los clusters en la representación ofrece también información muy significativa sobre la estructura de los géneros clásicos. Así, si seguimos un eje horizontal imaginario es fácil observar que a la izquierda del gráfico se sitúan los que cuentan 


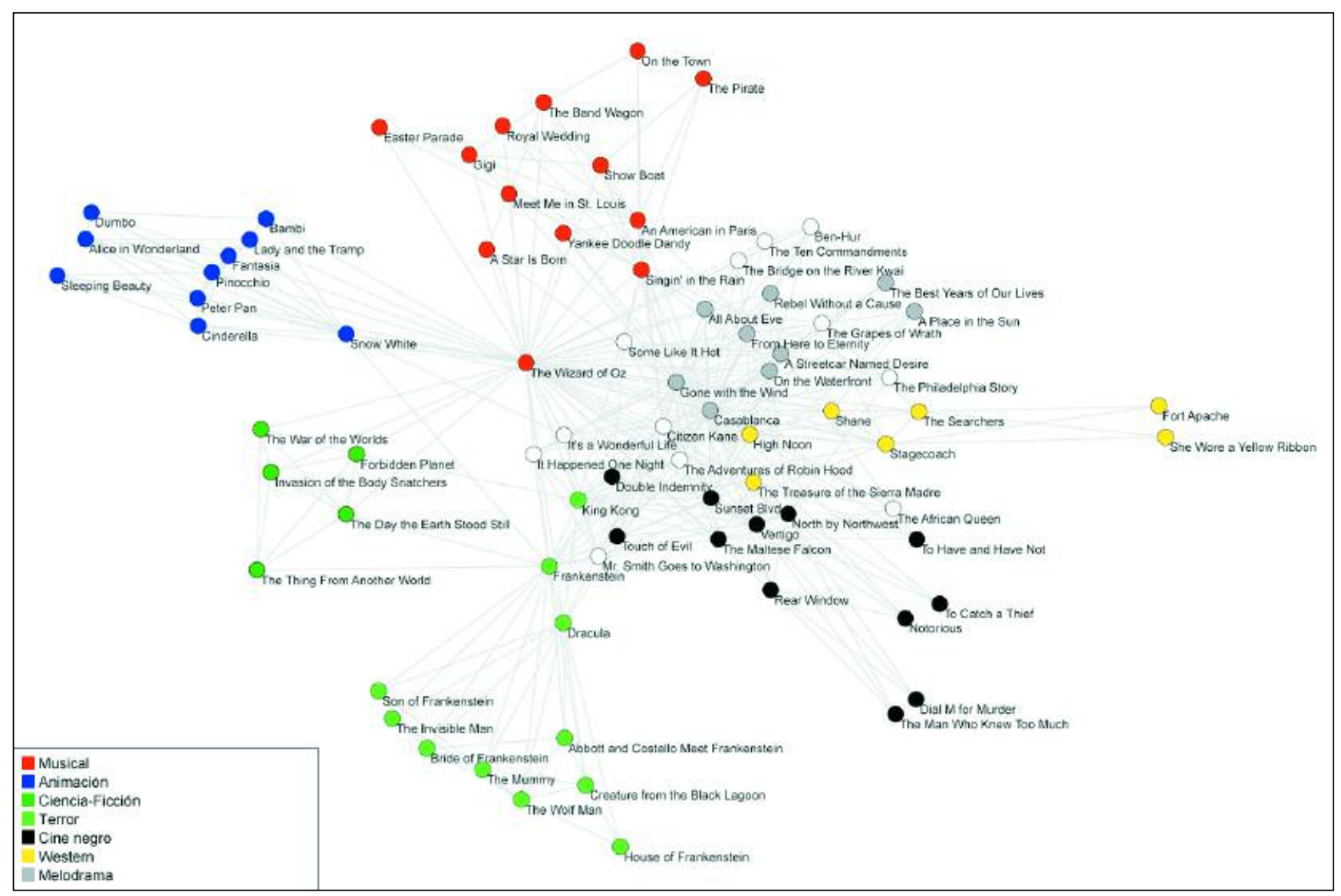

Figura 2

con una vocación "fantástica" (terror, ciencia-ficción, animación y musical), mientras que a la derecha quedan los que son más realistas (melodrama, western y cine negro); entendiendo por "realista" no tanto la vocación de reflejar fielmente la realidad (incluso en el plano argumental, todos ellos siguen patrones bien codificados) como de someterse a las reglas lógicas que en ella rigen.

Asimismo, si trazamos un eje vertical resulta evidente que en la zona superior se agrupan los géneros que en Hollywood tradicionalmente se han considerado women's pictures (el musical y el melodrama), junto con el cine de animación, dirigido al público infantil. En la parte inferior quedarían los que cuentan con un contenido más violento y un tono pesimista: el western, el cine negro, el terror y la incipiente ciencia-ficción.

Las posiciones centrales del gráfico están ocupadas por filmes que o bien no tienen una adscripción genérica clara o son influyentes en más de un género. Así sucede, por ejemplo, con la película que tiene un mayor índice de intermediación ${ }^{6}$, El mago de $\mathrm{Oz}$ (Fleming, 1939) que combina las aventuras destinadas a un público familiar (en línea con el cine de animación de Disney) con números musicales y una imaginería visual que ha tenido una gran influencia en el cine fantástico. Igualmente ocurre con películas como Casa- blanca (Curtiz, 1942) o Ciudadano Kane (Welles, 1941) que en su estilo visual, y en parte en su argumento, anticipan el cine negro, aunque incorporando elementos más realistas y dramáticos; o King Kong (Cooper; Schoedsack, 1933) que comparte elementos del cine de terror y de aventuras.

Factores como la presencia de determinadas estrellas o la autoría dan cuenta de algunos desplazamientos y agrupaciones en apariencia irregulares. Así, por ejemplo, la comedia La reina de África (1951) se sitúa en posiciones próximas al cine negro por la doble presencia del director John Huston y el actor Humphrey Bogart, al igual que le ocurría a El tesoro de Sierra Madre. Algo parecido sucede con las tres películas de Frank Capra representadas (Sucedió una noche, Qué bello es vivir y Caballero sin espada), que se sitúan muy próximas entre sí en posiciones centrales del gráfico. Asimismo, las películas de Alfred Hitchcock representadas aparecen enlazadas entre sí en el área de thriller/cine negro.

En cualquier caso, la nítida presencia de una serie de géneros con un alto grado de especialización narrativa y estética no debe hacer olvidar que existen películas de las que no se puede dar cuenta únicamente con la teoría de géneros. En particular, el melodrama muestra una cierta dispersión, síntoma de unos códigos sintácticos y semánticos más laxos que los demás 
géneros. Asimismo, comedias como Historias de Filadelfia (Cukor, 1940) o Con faldas y a lo loco (Wilder, 1959), dramas históricos como Los diez mandamientos (DeMille, 1956) o Ben-Hur (Wyler, 1959), películas bélicas como El puente sobre el río Kwai (Lean, 1957) o las comedias de trasfondo social de Frank Capra no encuentran un acomodo claro en la representación. Así pues, resulta evidente que a pesar de su indudable utilidad el sistema de los géneros no puede ser el único modelo para analizar una realidad tan compleja como el Hollywood clásico.

\section{Conclusiones}

El análisis de la intertextualidad cinematográfica es crucial para entender buena parte del cine contemporáneo, sobre todo en Occidente. A través del estudio de los procesos de derivación y contagio de ideas dentro de un medio tan autorreflexivo como el cinematográfico se ponen de manifiesto patrones de indudable utilidad para sistematizar el análisis de la producción y el consumo de textos fílmicos.

En particular, el análisis cuantitativo de las citas cinematográficas se muestra como una herramienta eficaz para contrastar las conclusiones alcanzadas desde acercamientos cualitativos y para mediar en debates abiertos. Este trabajo demuestra que la aplicación de técnicas de visualización a los datos obtenidos del recuento de co-citaciones cinematográficas pone de manifiesto estructuras subyacentes de influencia desde la percepción de los propios autores, lo que supone un avance sustancial para la teoría de géneros.

No obstante, los posibles usos de esta técnica no se limitan al ámbito académico. Por ejemplo, las visualizaciones generadas pueden emplearse como interfaz para facilitar la recuperación de información por medio de browsing en videotecas o bases de datos cinematográficas, o bien para complementar los mecanismos de recomendación en tiendas virtuales o similares.

Evidentemente, esta investigación no agota las posibilidades que el análisis cuantitativo de citas abre en el ámbito de los estudios cinematográficos. Otras técnicas como el análisis de co-citación de autores, medidas como la identidad de citas (vínculos de un texto/autor con aquellos a los que cita) y perspectivas como el análisis diacrónico, que muestra el desplazamiento de los elementos a través de sucesivas representaciones, pueden aportar información de gran interés para esta disciplina.

\section{Agradecimientos}

La investigación en la que se basa este artículo fue desarrollada bajo la supervisión del profesor Carlos OImeda Gómez, de la Universidad Carlos III de Madrid.

\section{Notas}

1. MDS es un conjunto de técnicas estadísticas que permite explorar similitudes y diferencias entre items o individuos con varias dimensiones o variables. Los algoritmos de MDS parten de una matriz (evidentemente, multidimensional) con cada item en filas y columnas; luego se asigna un lugar a cada item en un espacio de sólo 2 ó 3 dimensiones que permite poderlos visualizar.

2. Música diegética es la que proviene de fuentes que el espectador puede identificar en la película que está viendo (instrumentos, una radio, etc.).

3. Consultado en: 01-04-06.

http://www.imdb.com

4. La versión de la lista empleada se descargó de $I M D b$ el 25 de enero de 2006. Las listas fuente de la base de datos, actualizadas periódicamente, se pueden descargar desde la web. Consultado en: 01-04-06.

http://www.imdb.com/interfaces

5. Consultado en: 01-04-06.

http://vlado.fmf.uni-lj.si/pub/networks/pajek/

6. La intermediación es una medida de influencia que da cuenta de la proporción de rutas críticas entre nodos (las más cortas) que pasan por un determinado nodo.

\section{Bibliografía}

Altman, Rick. «A semantic/syntactic approach to film genre». En: Grant, Barry Keith (ed.). Film genre reader III. Austin: University of Texas Press, 2003, pp. 243-261. Isbn 0292701853.

Carroll, Noël. «The future of allusion: Hollywood in the seventies (and beyond)». En: Carroll, Noël. Interpreting the moving image. Cambridge; New York: Cambridge University Press, 1998, pp. 240-264. Isbn 0521589703 .

Coursodon, Jean Pierre. «La evolución de los géneros». En: Rimbau, Esteve; Torreiro, Casimiro (eds.). Historia general del cine: Volumen VIII. Madrid: Cátedra, 1996, pp. 225-307. Isbn 8437614422.

Garfield, Eugene. «Introducing the ISI Atlas of science: biochemistry and molecular biology, 1978-1980». En: Current contents, 1981, v. 42, pp. $5-13$.

Genette, Gerard. Palimpsestos: la literatura en segundo grado. Madrid: Taurus, 1989. Isbn 8430621954.

Iampolski, Mijaíl. La teoría de la intertextualidad y el cine. Valencia: Episteme, 1996. Isbn 8489447519.

Small, Henry; Griffith, Bellver C. «The structure of scientific literatures I: identifying and graphing specialities». En: Social studies, 1974, v. 4, pp. 17-40.

Staiger, Janet. «Hybrid or inbred: the purity hypothesis and Hollywood genre history». En: Grant, Barry Keith (ed.). Film genre reader III. Austin: University of Texas Press, 2003, pp. 185-202. Isbn 0292701853.

Stam, Robert. Teorías del cine: una introducción. Barcelona: Paidós Ibérica, 2001. Isbn 8449310636.

Walter, Jörg A. «H-Mds: a new approach for interactive visualization with multidimensional scaling in the hyperbolic space». En: Information systems, 2004, v. 29, n. 4, pp. 273-292.

White, Howard D.; McCain, Katherine W. «Visualizing a discipline: an author co-citation analysis of information science, 1972-1995». En: Journal of the American Society for Information Science, 1998, v. 49, n. 4, pp. 327-355.

Carlos Leal Zubiete, Sociedad Andaluza para el Desarrollo de la Sociedad de la Información. carlos.leal.ext@juntadeandalucia.es 